\title{
Single pellet combustion of sewage sludge and agricultural residues with a focus on phosphorus
}

Gustav Häggström, Thomas Karl Hannl, Ali Hedayati, Matthias Kuba, Nils Skoglund, Marcus Öhman

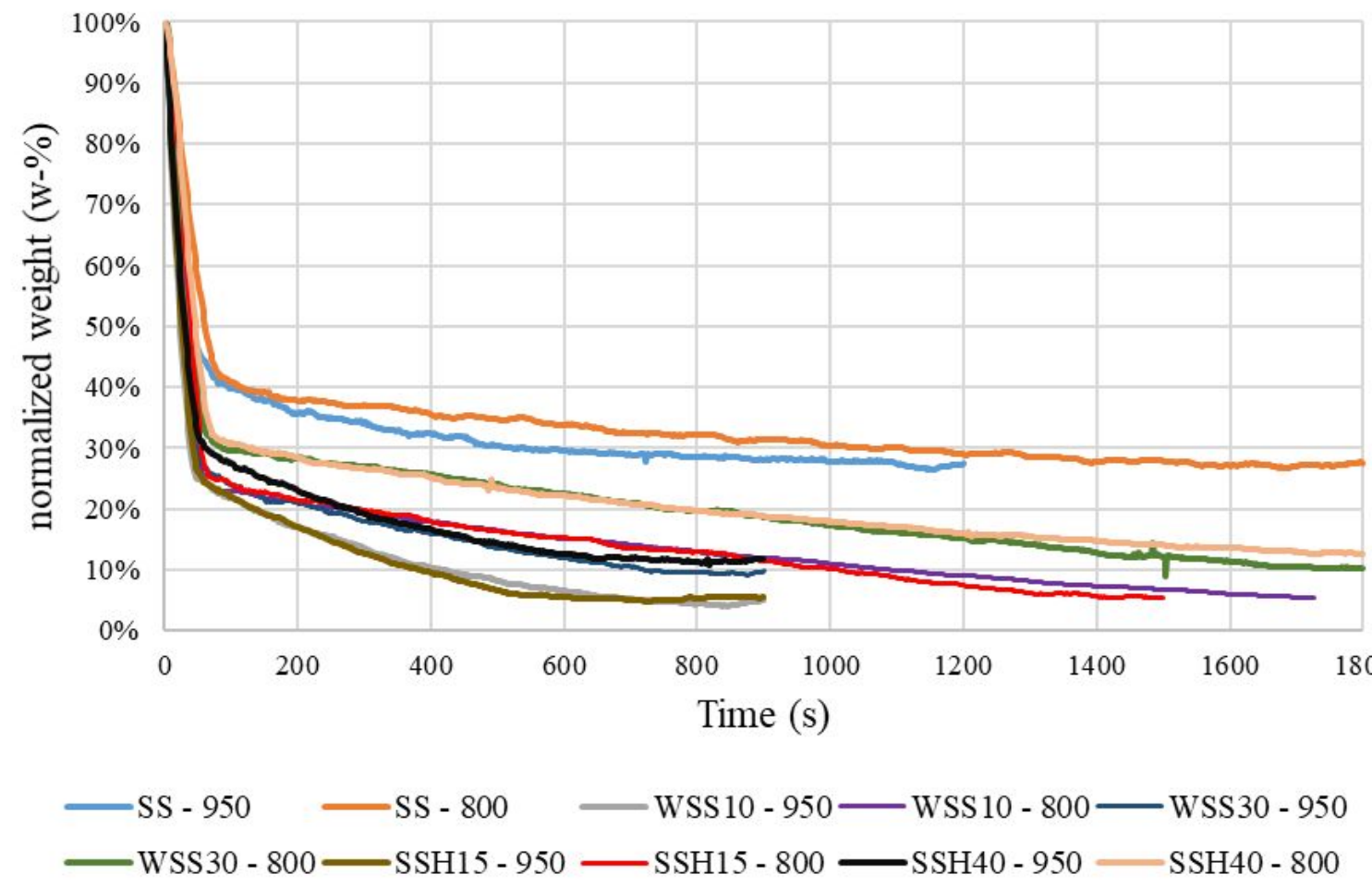

Figure S1. Representative thermogravimetric analysis (TGA) curves for SS, WSS10, WSS30, SHS15 and SHS40 performed at isothermal temperatures of 800 and $950{ }^{\circ} \mathrm{C}$. 

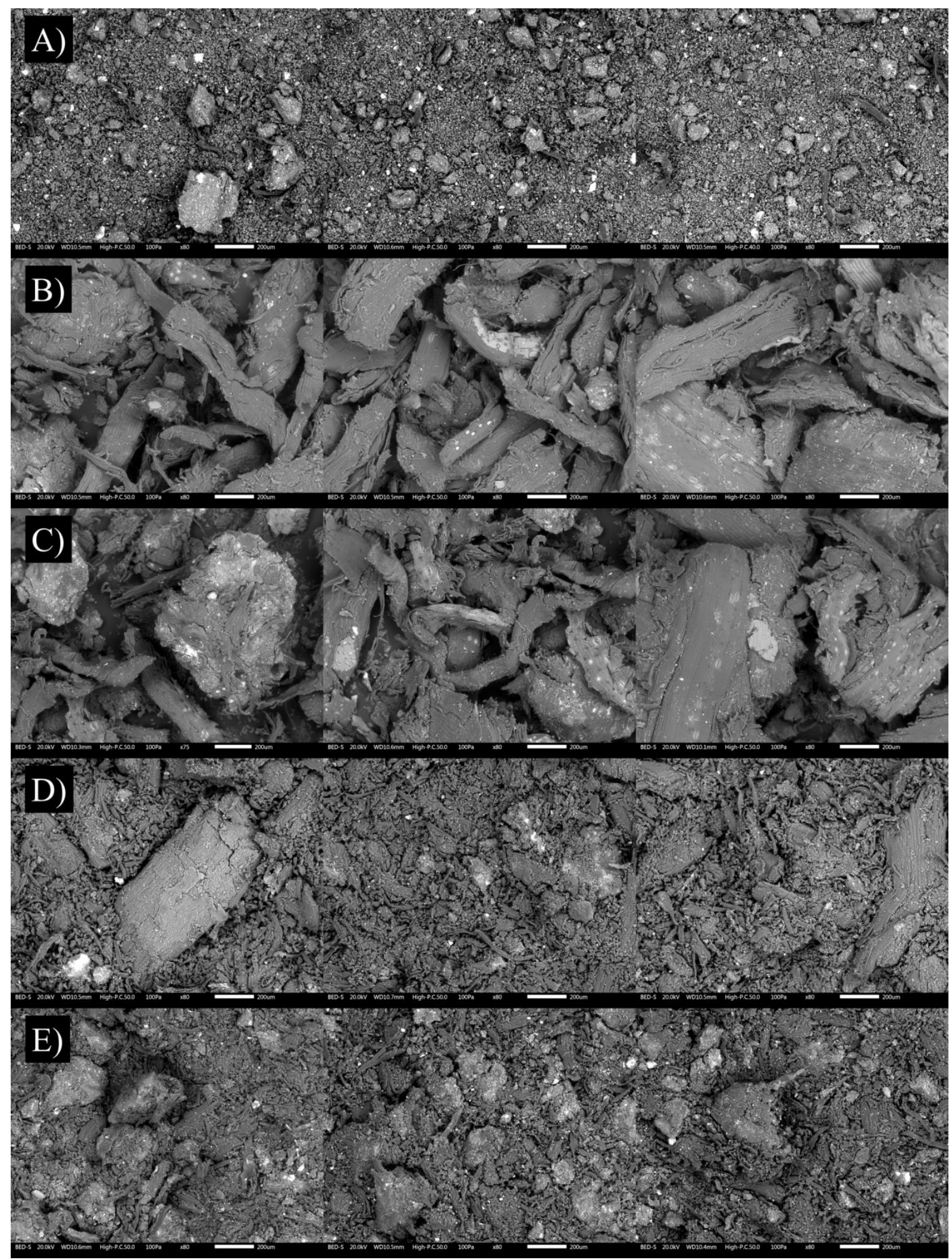

Figure S2. BSE-SEM micrographs of the raw fuels. The reader is referred to Figure 2 of the paper for interpretation of morphological features. A) SS, B) WSS10, C) WSS30, D) SHS15 \& E) SHS40 